\title{
Constraints on Neutrino Masses from Baryon Acoustic Oscillation Measurements
}

\author{
B. Hoeneisen \\ Universidad San Francisco de Quito, Quito, Ecuador \\ Email:bruce1@fnal.gov
}

How to cite this paper: Hoeneisen, B. (2018) Constraints on Neutrino Masses from Baryon Acoustic Oscillation Measurements. International Journal of Astronomy and Astrophysics, 8, 1-5.

https://doi.org/10.4236/ijaa.2018.81001

Received: December 12, 2017

Accepted: February 6, 2018

Published: February 9, 2018

Copyright (C) 2018 by author and Scientific Research Publishing Inc. This work is licensed under the Creative Commons Attribution International License (CC BY 4.0)

http://creativecommons.org/licenses/by/4.0/

\begin{abstract}
From 21 independent Baryon Acoustic Oscillation (BAO) measurements we obtain the following sum of masses of active Dirac or Majorana neutrinos: $\sum m_{v}=0.711-0.335 \cdot \delta h+0.050 \cdot \delta b \pm 0.063 \mathrm{eV}$, where $\delta h \equiv(h-0.678) / 0.009$ and $\delta b \equiv\left(\Omega_{b} h^{2}-0.02226\right) / 0.00023$. This result may be combined with independent measurements that constrain the parameters $\sum m_{v}, h$, and $\Omega_{b} h^{2}$. For $\delta h= \pm 1$ and $\delta b= \pm 1$, we obtain $m_{v}<0.43 \mathrm{eV}$ at $95 \%$ confidence.
\end{abstract}

\section{Keywords}

Neutrino Mass, Baryon Accoustic Oscillations, Cosmology

\section{Introduction}

We extend the analysis presented in "Study of baryon acoustic oscillations with SDSS DR13 data and measurements of $\Omega_{k}$ and $\Omega_{\mathrm{DE}}(a)$ " [1] to include neutrino masses. The present analysis has three steps: 1) we calculate the distance of propagation $r_{s}$, in units of $c / H_{0}$, referred to the present time, of sound waves in the photon-electron-baryon plasma until decoupling by numerical integration of Equation (16) and Equation (17) of Ref. [1];2) we fit the Friedmann equation of evolution of the universe to 21 independent Baryon Acoustic Oscillation (BAO) distance measurements listed in [1] used as uncalibrated standard rulers and obtain the length $d$ of these rulers, in units of $c / H_{0}$, referred to the present time; and 3) we set

$$
r_{s}=d
$$

to constrain the sum of neutrino masses $\sum m_{v} . c$ is the speed of light, and $H_{0} \equiv 100 \mathrm{hm} \cdot \mathrm{s}^{-1} \cdot \mathrm{Mpc}^{-1}$ is the present day Hubble expansion parameter. 


\section{Constraints on Neutrino Masses}

The main body of this article assumes: 1) flat space, i.e. $\Omega_{k}=0$, and 2) constant dark energy density relative to the critical density, i.e. $\Omega_{\mathrm{DE}}$ independent of the expansion parameter $a$. These constraints are in agreement with all observations to date [1] [2]. Results without these constraints are presented in Appendix 1. Results with partial data sets are presented in Appendix 2.

To be specific we consider three active neutrino flavors with three eigenstates with nearly the same mass $m_{v}$, so $\sum m_{v}=3 m_{v}$. This is a useful scenario to consider since our current limits on $m_{v}^{2}$ are much larger than the mass-squared-differences $\Delta m^{2}$ and $\Delta m_{21}^{2}$ obtained from neutrino oscillations [2]. These neutrinos become non-relativistic at a neutrino temperature $T_{v}=m_{v} / 3.15$ or a photon temperature $T=m_{v}(11 / 4)^{1 / 3} / 3.15$. The corresponding expansion parameter is $a_{v}=T_{0} / T=5.28 \times 10^{-4}\left(1 \mathrm{eV} / m_{v}\right)$.

The matter density relative to the present critical density is $\Omega_{m} / a^{3}$ for $a>a_{v} . \Omega_{m}$ includes the density $\Omega_{v}=h^{-2} \sum m_{v} / 94 \mathrm{eV}$ of Dirac or Majorana neutrinos that are non-relativistic today. Note that for Dirac neutrinos we are considering the scenario in which right-handed neutrinos and left-handed anti-neutrinos are sterile and never achieved thermal equilibrium. Our results can be amended for other specific scenarios. For $a<a_{v}$ we take the matter density to be $\left(\Omega_{m}-\Omega_{v}\right) / a^{3}$. The radiation density is $\Omega_{\gamma} N_{\text {eq }} /\left(2 a^{4}\right)$ for $a<a_{v}$, where $N_{\mathrm{eq}}=3.36$ for three flavors of Dirac (mostly) left-handed neutrinos and right-handed anti-neutrinos. We also take $N_{\text {eq }}=3.36$ for three active flavors of Majorana left-handed and right-handed neutrinos. For $a>a_{v}$, we take the radiation density to be $\left(\Omega_{\gamma} N_{e q} / 2-a_{v} \Omega_{v}\right) / a^{4}=\Omega_{\gamma} / a^{4}$. The present density of photons relative to the critical density is $\Omega_{\gamma}=2.473 \times 10^{-5} \mathrm{~h}^{-2} \quad$ [2].

The data used to obtain $d$ are 18 independent BAO distance measurements with Sloan Digital Sky Survey (SDSS) data release DR13 galaxies in the redshift range $z=0.1$ to 0.7 [3] [4] [5] summarized in Table 3 of [1], two BAO distance measurements in the Lyman-alpha forest $(\operatorname{Ly} \alpha)$ at $z=2.36$ (cross-correlation [6]) and $z=2.34$ (auto-correlation [7]) summarized in Section 6 of [1], and the Cosmic Microwave Background (CMB) correlation angle $\theta_{\mathrm{MC}}=0.010410 \pm 0.000005$ [2] [8], used as an uncalibrated standard ruler. Note that the correlation angle $\theta_{\mathrm{MC}}$ is also determined by BAO. These 21 independent $\mathrm{BAO}$ measurements and full details of the fitting method are presented in [1].

As a reference we take

$$
h=0.678 \pm 0.009, \quad \Omega_{b} h^{2}=0.02226 \pm 0.00023
$$

(at 68\% confidence) from "Planck TT + low P + lensing" data (that does not contain BAO information) [2]. $\Omega_{b}$ is the present density of baryons relative to the critical density.

Due to correlations and non-linearities we obtain our final result (Equation (9) below) with a global fit. The following equations are included to illustrate the dependence of $r_{s}$ and $d$ on the cosmological parameters $h, \Omega_{b} h^{2}$ and $\sum m_{v}$ 
in limited ranges of interest. Integrating the comoving sound speed of the photon-baryon-electron plasma until $a_{\mathrm{dec}}=1 /\left(1+z_{\mathrm{dec}}\right)$ with $z_{\mathrm{dec}}=1089.9 \pm 0.4$ [2] we obtain

$$
r_{s} \approx 0.0339 \times A \times\left(\frac{0.28}{\Omega_{m}}\right)^{0.24}
$$

with

$$
A \approx 0.990+0.007 \cdot \delta h-0.001 \cdot \delta b+0.020 \cdot \frac{\sum m_{v}}{1 \mathrm{eV}},
$$

where

$$
\begin{gathered}
\delta h \equiv(h-0.678) / 0.009, \\
\delta b \equiv\left(\Omega_{b} h^{2}-0.02226\right) / 0.00023 .
\end{gathered}
$$

To obtain $d$ we minimize the $\chi^{2}$ with 21 terms, corresponding to the 21 BAO observables, with respect to $\Omega_{\mathrm{DE}}$ and $d$, and obtain $\Omega_{\mathrm{DE}}=0.718 \pm 0.003$ and

$$
d \approx 0.0340 \pm 0.0002 \text {, }
$$

with $\chi^{2}$ per degree of freedom 19.8/19, and correlation coefficient 0.989 between $\Omega_{\mathrm{DE}}$ and $d$ (this high correlation coefficient is due to the high precision of $\theta_{\mathrm{MC}}$ ). Setting $r_{s}=d$ we obtain

$$
\sum m_{v} \approx 0.73-0.35 \cdot \delta h+0.05 \cdot \delta b \pm 0.15 \mathrm{eV} .
$$

A more precise result is obtained with a global fit by minimizing the $\chi^{2}$ with 21 terms varying $\Omega_{\mathrm{DE}}$ and $\sum m_{v}$ directly. We obtain $\Omega_{\mathrm{DE}}=0.7175 \pm 0.0023$ and

$$
\sum m_{v}=0.711-0.335 \cdot \delta h+0.050 \cdot \delta b \pm 0.063 \mathrm{eV},
$$

with $\chi^{2} /$ d.f. $=19.9 / 19$, and correlation coefficient 0.924 between $\Omega_{\mathrm{DE}}$ and $\sum m_{v}$. This is our main result. Equation (9) is obtained from BAO measurements alone, and is written in a way that can be combined with independent constraints on the cosmological parameters $\sum m_{v}, h$ and $\Omega_{b} h^{2}$, such as measurements of the power spectrum of density fluctuations $P(k)$, the $\mathrm{CMB}$, and direct measurements of the Hubble parameter.

Setting $\delta h= \pm 1$ and $\delta b= \pm 1$ we obtain the following upper bound on the mass of active neutrinos $m_{v}=\frac{1}{3} \sum m_{v}$ :

$$
m_{v}<0.43 \mathrm{eV} \text { at } 95 \% \text { confidence. }
$$

\section{References}

[1] Hoeneisen, B. (2017) Study of Baryon Acoustic Oscillations with SDSS DR13 Data and Measurements of $\Omega_{k}$ and $\Omega_{\mathrm{DE}}(a)$. International Journal of Astronomy and Astrophysics, 7, 11-27. https://doi.org/10.4236/ijaa.2017.71002

[2] Patrignani, C., et al. (2016) Review of Particle Physics. Chinese Physics C, 40, Article ID: 100001. 
[3] Albareti, F.D., et al. (2016) The Thirteenth Data Release of the Sloan Digital Sky Survey: First Spectroscopic Data from the SDSS-IV Survey Mapping Nearby Galaxies at Apache Point Observatory. SDSS Collaboration, arXiv:1608.02013.

[4] Dawson, K.S., Schlegel, D.J., Ahn, C.P., et al. (2013) The Baryon Oscillation Spectroscopic Survey of SDSS-III. Astronomical Journal, 145, 10.

https://doi.org/10.1088/0004-6256/145/1/10

[5] Dawson, K.S., Kneib, J.-P., Percival, W.J., et al. (2016) The SDSS-IV Extended Baryon Oscillation Spectroscopic Survey: Overview and Early Data. Astronomical Journal, 151, 44. https://doi.org/10.3847/0004-6256/151/2/44

[6] Font-Ribera, A., et al. (2014) Quasar-Lyman $\alpha$ Forest Cross-Correlation from BOSS DR11: Baryon Acoustic Oscillations. arXiv:1311.1767.

https://doi.org/10.1088/1475-7516/2014/05/027

[7] Delubac, T., et al. (2014) Baryon Acoustic Oscillations in the Ly $\alpha$ Forest of BOSS DR11 Quasars. arXiv:1404.1801v2.

[8] Planck Collaboration (2015) Results XIII, Astron. \& Astrophys. Submitted, arXiv:1502.01589v2. 


\section{Appendix}

Appendix 1. Removing constraints

Freeing $\Omega_{k}$ and keeping $\Omega_{\mathrm{DE}}$ constant we obtain $\Omega_{k}=-0.003 \pm 0.006$, $\Omega_{\mathrm{DE}}+2.2 \Omega_{k}=0.719 \pm 0.003$, and

$$
\sum m_{v}=0.623-0.334 \cdot \delta h+0.050 \cdot \delta b \pm 0.191 \mathrm{eV},
$$

with $\chi^{2} /$ d.f. $=19.6 / 18$.

Fixing $\Omega_{k}=0$ and letting $\Omega_{\mathrm{DE}}(a)=\Omega_{\mathrm{DE}} \cdot\left\{1+w_{a} \cdot(1-a)\right\} \quad$ we obtain $\Omega_{\mathrm{DE}}=0.716 \pm 0.004, w_{a}=0.064 \pm 0.148$, and

$$
\sum m_{v}=0.603-0.349 \cdot \delta h+0.052 \cdot \delta b \pm 0.257 \mathrm{eV},
$$

with $\chi^{2} /$ d.f. $=19.7 / 18$.

Freeing $\Omega_{k}$ and letting $\Omega_{\mathrm{DE}}(a)=\Omega_{\mathrm{DE}} \cdot\left\{1+w_{a} \cdot(1-a)\right\} \quad$ we obtain $\Omega_{k}=-0.008 \pm 0.004, \Omega_{\mathrm{DE}}+2.2 \Omega_{k}=0.718 \pm 0.004, w_{a}=0.227 \pm 0.069$, and

$$
0<\sum m_{v}=-0.388-0.350 \cdot \delta h+0.050 \cdot \delta b \pm 0.830 \mathrm{eV},
$$

with $\chi^{2} /$ d.f. $=17.8 / 17$.

Appendix 2. Removing data.

In this Appendix we apply the constraints $\Omega_{k}=0$ and $\Omega_{\mathrm{DE}}$ constant. Removing the measurement of $\theta_{\mathrm{MC}}$ we obtain $\Omega_{\mathrm{DE}}=0.722 \pm 0.011$ and

$$
\sum m_{v}=0.579-0.333 \cdot \delta h+0.049 \cdot \delta b \pm 0.285 \mathrm{eV},
$$

with $\chi^{2} /$ d.f. $=19.7 / 18$.

Removing the measurement of $\theta_{\mathrm{MC}}$ and the two Ly $\alpha$ measurements we obtain $\Omega_{\mathrm{DE}}=0.716 \pm 0.014$ and

$$
\sum m_{v}=0.743-0.330 \cdot \delta h+0.049 \cdot \delta b \pm 0.366 \mathrm{eV},
$$

with $\chi^{2} /$ d.f. $=11.2 / 16$.

Keeping only the measurement of $\theta_{\mathrm{MC}}$ we need to fix $\Omega_{\mathrm{DE}}$ in order to get zero degrees of freedom and have a unique solution. The best way to fix $\Omega_{\mathrm{DE}}$ is with BAO measurements, and that is the purpose of the present study. 